\title{
CONSTRUCTION AND INFERENCES OF THE EFFICIENT FRONTIER IN ELLIPTICAL MODELS
}

\author{
Taras Bodnar* and Arjun K. Gupta**
}

\begin{abstract}
In this paper, we construct a confidence region for the efficient frontier assuming the asset returns to be matrix elliptically contoured distributed. Our results extend the findings of Bodnar and Schmid (2009) to the non-normal distributed asset returns. In order to correct the overoptimism of the sample efficient frontier documented in Siegel and Woodgate (2007), the unbiased estimator of the efficient frontier is suggested. Moreover, we derive an exact overall $F$-test for the efficient frontier in elliptical models.
\end{abstract}

Key words and phrases: Efficient frontier, mean-variance portfolio, parameter uncertainty, interval estimation, matrix variate elliptically contoured distribution, matrix variate $t$-distribution.

\section{Introduction}

In order to construct an optimal portfolio Markowitz (1952) proposed to choose the portfolio with the smallest risk for a given level of average portfolio return. Merton (1972) showed that the set of all of these optimal portfolios lies on a parabola in the mean-variance space, the so-called efficient frontier. This parabola is determined by three characteristics. The first two define the location of the vertex, while the third one is the slope parameter. Assuming the asset returns to be independently and normally distributed, Jobson (1991) obtained an asymptotic confidence region of the efficient frontier, while Bodnar and Schmid (2009) derived an exact joint confidence set of the three parameters of the efficient frontier. This set is used to determine a region in the mean-variance space where all optimal portfolios lie with a given probability $(1-\alpha)$.

Because the parameters of the efficient frontier are unknown quantities, the investor cannot construct the efficient frontier. Usually, the sample efficient frontier is used instead of the population efficient frontier (see, e.g. Bodnar and Schmid (2008b), Kan and Smith (2008)), which is obtained by replacing the unknown parameters of the asset returns distribution with the corresponding sample counterparts. However, Basak et al. (2005) and Siegel and Woodgate (2007) showed that the sample efficient frontier is overoptimistic and overestimates the true location of the efficient frontier in the mean-variance space. In order to correct this overoptimism Kan and Smith (2008) suggested an improved estimator of the efficient frontier, while Bodnar and Bodnar (2009) derived the

\footnotetext{
Received December 20, 2008. Revised April 17, 2009. Accepted July 17, 2009.

*Department of Statistics, European University Viadrina, PO Box 1786, 15207 Frankfurt (Oder), Germany.

**Department of Mathematics and Statistics, Bowling Green State University, Bowling Green, OH 43403, U.S.A.
} 
unbiased estimator of the efficient frontier.

In the above cited papers, the assumption of independence and normality is maintained. However, these assumptions might not be appropriate in many situations of practical interest. Many authors have shown that the distribution of daily stock returns is heavy tailed (e.g., Fama (1965), Markowitz (1991), Mittnik and Rachev (1993), Chen et al. (2003)). The assumption of independent asset returns turns out to be questionable, too. Numerous studies have shown that the returns are uncorrelated but not independent (cf. Engle (1982), Bollerslev (1986), Nelson (1991)).

In the present paper, a weaker assumption is imposed on the distribution of the asset returns. We assume that the matrix of returns follows a matrix elliptically contoured distribution (see, e.g. Fang and Zhang (1990), Gupta and Varga (1993)). This class covers a wide range of distributions, e.g., the normal distribution, the mixture of normal distributions, Pearson types II and VII distributions, the multivariate $t$-distribution, the logistic distribution, scale distributions (Gupta and Varga (1993)). Elliptical distributions, whose contours of equal density have the same elliptical shape as the normal, provide attractive and intuitive alternatives to the multivariate normality. Elliptically contoured distributions have already been discussed in financial literature. For instance, Owen and Rabinovitch (1983) extended Tobin's separation theorem, Bawa's rules of ordering certain prospects to elliptically contoured distributions. Chamberlain (1983) showed that elliptical distributions imply mean-variance utility functions, while Berk (1997) argued that one of the necessary conditions for the capital asset pricing model (CAPM) is an elliptical distribution for the asset returns. Zhou (1993) extended findings of Gibbons et al. (1989) by applying their test of the validity of the CAPM to elliptically distributed returns. A further test for the CAPM under elliptical assumptions is proposed by Hodgson et al. (2002). Assuming the matrix of asset returns to be matrix elliptically contoured distributed, Bodnar and Schmid (2007) derived an exact test on the global minimum variance.

The rest of the paper is organized as follows. In the next section, we present a definition of the elliptically contoured distribution and derive an expression for the efficient frontier in elliptical models. Main results are given in Section 3. In Theorem 1, the exact distribution of the estimated characteristics of the efficient frontier are derived assuming the asset returns to follow a matrix variate mixture of normal distributions. In Section 3.2, the confidence region of the efficient frontier is presented, while the unbiased estimator of the efficient frontier is given in Section 3.3. In Section 3.4, we show that the overall F-test derived by Bodnar and Bodnar (2009) is distribution free in the class of matrix elliptically contoured distributions. In Section 4, a confidence region of the efficient frontier is determined in a practical relevant situation assuming the asset returns to be matrix variate $t$-distributed with 5 and 15 degrees of freedom. 


\section{Derivation of the efficient frontier}

In this section, we derive an expression for the efficient frontier assuming that the asset returns are elliptically contoured distributed. A random vector $\boldsymbol{x}$ is elliptically contoured distributed with the location parameter $\boldsymbol{\mu}$ and the dispersion matrix $\boldsymbol{\Sigma}$ if it has the following stochastic representation (see, e.g. Fang and Zhang (1990, p. 65))

$$
\boldsymbol{x} \stackrel{d}{=} \boldsymbol{\mu}+\tilde{r} \boldsymbol{\Sigma}^{1 / 2} \boldsymbol{u}
$$

where $\boldsymbol{u}$ is uniformly distributed on the unit sphere in $\mathbb{R}^{k}$ and the generating variable $\tilde{r}$ is independent of $\boldsymbol{u}$. The symbol $\stackrel{d}{=}$ denotes the equality in distribution. When the density of $\boldsymbol{x}$ exists, it has the following form

$$
f(\boldsymbol{x})=|\boldsymbol{\Sigma}|^{-1 / 2} g\left((\boldsymbol{x}-\boldsymbol{\mu})^{\prime} \boldsymbol{\Sigma}^{-1}(\boldsymbol{x}-\boldsymbol{\mu})\right),
$$

where $g($.$) is the density generator of the random vector \boldsymbol{x}$.

We derive an expression for the efficient frontier using the formulas for the expected return and the variance of the optimal portfolio in the sense of maximizing the expected utility function (EU portfolio). Let $\boldsymbol{w}=\left(w_{1}, \ldots, w_{k}\right)^{\prime}$ denote the vector of portfolio weights, i.e. $w_{i}$ is the part of the investor's wealth invested into the $i$ th asset. Then the expected return of the portfolio with the weight vector $\boldsymbol{w}$ is given by $R_{p}=\boldsymbol{w}^{\prime} \mathrm{E}(\boldsymbol{x})=\boldsymbol{w}^{\prime} \boldsymbol{\mu}$, while its variance is $V_{p}=\boldsymbol{w}^{\prime} \operatorname{Var}(\boldsymbol{x}) \boldsymbol{w}=\frac{E\left(\tilde{r}^{2}\right)}{k} \boldsymbol{w}^{\prime} \boldsymbol{\Sigma} \boldsymbol{w}$. The weights of the EU portfolio are obtained by maximizing

$$
R_{p}-\frac{\gamma}{2} V_{p}=\boldsymbol{w}^{\prime} \boldsymbol{\mu}-\frac{\gamma}{2} \frac{E\left(\tilde{r}^{2}\right)}{k} \boldsymbol{w}^{\prime} \boldsymbol{\Sigma} \boldsymbol{w}
$$

under the constraint $\boldsymbol{w}^{\prime} \mathbf{1}=1$. There $\gamma>0$ is the coefficient of the investor's risk aversion. The solution of (2.2) is given by

$$
\boldsymbol{w}_{E U}=\frac{\boldsymbol{\Sigma}^{-1} \mathbf{1}}{\mathbf{1}^{\prime} \boldsymbol{\Sigma}^{-1} \mathbf{1}}+\tilde{\gamma}^{-1} \boldsymbol{Q} \boldsymbol{\mu} \quad \text { with } \quad \boldsymbol{Q}=\boldsymbol{\Sigma}^{-1}-\frac{\boldsymbol{\Sigma}^{-1} \mathbf{1}^{\prime} \boldsymbol{\Sigma}^{-1}}{\mathbf{1}^{\prime} \boldsymbol{\Sigma}^{-1} \mathbf{1}}
$$

where $\tilde{\gamma}=\gamma E\left(\tilde{r}^{2}\right) / k$. Using the weights (2.3), the expected return and the variance of the EU portfolio are obtained as

$$
R_{E U}=\frac{\boldsymbol{\mu}^{\prime} \boldsymbol{\Sigma}^{-1} \mathbf{1}}{\mathbf{1}^{\prime} \boldsymbol{\Sigma}^{-1} \mathbf{1}}+\tilde{\gamma}^{-1} \boldsymbol{\mu}^{\prime} \boldsymbol{Q} \boldsymbol{\mu}=R_{G M V}+\tilde{\gamma}^{-1} s
$$

and

$$
V_{E U}=\frac{E\left(\tilde{r}^{2}\right)}{k} \frac{1}{\mathbf{1}^{\prime} \boldsymbol{\Sigma}^{-1} \mathbf{1}}+\tilde{\gamma}^{-2} \frac{E\left(\tilde{r}^{2}\right)}{k} \boldsymbol{\mu}^{\prime} \boldsymbol{Q} \boldsymbol{\mu}=\frac{E\left(\tilde{r}^{2}\right)}{k} V_{G M V}+\tilde{\gamma}^{-2} \frac{E\left(\tilde{r}^{2}\right)}{k} s
$$

where

$$
R_{G M V}=\frac{\mathbf{1}^{\prime} \boldsymbol{\Sigma}^{-1} \boldsymbol{\mu}}{\mathbf{1}^{\prime} \boldsymbol{\Sigma}^{-1} \mathbf{1}}, \quad \text { and } \quad V_{G M V}=\frac{1}{\mathbf{1}^{\prime} \boldsymbol{\Sigma}^{-1} \mathbf{1}}
$$


are the expected return and the variance of the global minimum variance portfolio (GMV portfolio) and $s=\boldsymbol{\mu}^{\prime} \boldsymbol{Q} \boldsymbol{\mu}$. The GMV portfolio is a special case of the EU portfolio that corresponds to the case of the fully risk averse investor, i.e. $\gamma=\infty$.

Equations (2.4) and (2.5) are considered as the parametric equations of the efficient frontier. Solving (2.4) and (2.5) with respect to $\tilde{\gamma}$, the efficient frontier is expressed as

$$
\left(R-R_{G M V}\right)^{2}=\frac{k}{E\left(\tilde{r}^{2}\right)} s\left(V-\frac{E\left(\tilde{r}^{2}\right)}{k} V_{G M V}\right) .
$$

From (2.7) we conclude that the efficient frontier depends on the asset returns distribution. If $E\left(\tilde{r}^{2}\right)>k$, then the risk of the investment is higher than in the normal case. Moreover, there is a decrease in the overall market profitability since in (2.7) the slope coefficient of the parabola is multiplied by $k / E\left(\tilde{r}^{2}\right)<1$. When $E\left(\tilde{r}^{2}\right) \rightarrow \infty$, the slope coefficient of the parabola tends to zero. In this case the efficient frontier degenerates into a straight line and the only efficient portfolio is the GMV portfolio.

The same result is obtained by considering $\tilde{\gamma}$. Note, that $\tilde{\gamma}$ can be considered as a coefficient of risk inversion in elliptical models. If $\gamma=\infty$ and $E\left(\tilde{r}^{2}\right)<\infty$, the EU portfolio transforms to the GMV portfolio. From the other side, if $E\left(\tilde{r}^{2}\right)=\infty$ there is no solution of the optimization problem since in this case we get that

$$
\left(R-R_{G M V}\right)^{2}=-s V_{G M V} .
$$

\section{Main results}

\subsection{Sample efficient frontier in elliptical models}

Let $\boldsymbol{x}_{1}, \ldots, \boldsymbol{x}_{n}$ be a sample of the asset returns. We assume that the asset returns follow a matrix variate mixture of normal distributions with mean vector $\boldsymbol{\mu}$ and dispersion matrix $\boldsymbol{\Sigma}$. Any mixture of normal distributions belongs to the family of elliptically contoured distributions with stochastic representation of $\boldsymbol{x}_{i}$ given by

$$
\boldsymbol{x}_{i} \stackrel{d}{=} \boldsymbol{\mu}+r \boldsymbol{\Sigma}^{1 / 2} \boldsymbol{z}_{i}
$$

where $\boldsymbol{z}_{i} \sim \mathcal{N}_{k}(\mathbf{0}, \boldsymbol{I})$ (k-dimensional normal distribution with mean vector $\mathbf{0}$ and covariance matrix $\boldsymbol{I})$ and $\boldsymbol{Z}=\left(\boldsymbol{z}_{1}, \ldots, \boldsymbol{z}_{n}\right)$ is independent of $r$. We denote this distribution by $\mathcal{M E D}(\boldsymbol{\mu}, \boldsymbol{\Sigma}, g()$.$) , where g($.$) is the so-called density generator$ which is fully determined by the distribution of $r$.

Note, that the asset returns are not assumed to be independently distributed in (3.1). The assumption of independence is replaced with a weaker one that the asset returns are uncorrelated. The random variable $r$ determines the tail behavior of the asset returns. The model (3.1) is in-line with the recent modeling of the daily behavior of the asset returns. The daily asset returns are heavy-tailed distributed and they are not independent (see, e.g. Engle (1982), Bollerslev (1986), Nelson (1991), Engle (2002)). 
Because $\boldsymbol{\mu}$ and $\boldsymbol{\Sigma}$ are unknown parameters of the asset returns distribution, the investor cannot use (2.7) to construct the efficient frontier. These quantities have to be estimated from the historical values of the asset returns before the efficient frontier is determined. We consider the sample estimators of these parameters given by

$$
\hat{\boldsymbol{\mu}}=\frac{1}{n} \sum_{j=1}^{n} \boldsymbol{x}_{j} \quad \text { and } \quad \hat{\boldsymbol{\Sigma}}=\frac{1}{n-1} \sum_{j=1}^{n}\left(\boldsymbol{x}_{j}-\hat{\boldsymbol{\mu}}\right)\left(\boldsymbol{x}_{j}-\hat{\boldsymbol{\mu}}\right)^{\prime} .
$$

Using $E\left(\tilde{r}^{2}\right) / k=E\left(r^{2}\right)$ and plugging (3.2) instead of $\boldsymbol{\mu}$ and $\boldsymbol{\Sigma}$ in (2.7), the sample efficient frontier is expressed as

$$
\left(R-\hat{R}_{G M V}\right)^{2}=\frac{1}{E\left(r^{2}\right)} \hat{s}\left(V-E\left(r^{2}\right) \hat{V}_{G M V}\right),
$$

where

$$
\hat{R}_{G M V}=\frac{\mathbf{1}^{\prime} \hat{\boldsymbol{\Sigma}}^{-1} \hat{\boldsymbol{\mu}}}{\mathbf{1}^{\prime} \hat{\boldsymbol{\Sigma}}^{-1} \mathbf{1}}, \quad \hat{V}_{G M V}=\frac{1}{\mathbf{1}^{\prime} \hat{\boldsymbol{\Sigma}}^{-1} \mathbf{1}}, \quad \text { and } \quad \hat{s}=\hat{\boldsymbol{\mu}}^{\prime} \hat{\boldsymbol{Q}} \hat{\boldsymbol{\mu}}
$$

with $\hat{\boldsymbol{Q}}=\hat{\boldsymbol{\Sigma}}^{-1}-\hat{\boldsymbol{\Sigma}}^{-1} \mathbf{1} \mathbf{1}^{\prime} \hat{\boldsymbol{\Sigma}}^{-1} / \mathbf{1}^{\prime} \hat{\mathbf{\Sigma}}^{-1} \mathbf{1}$.

In Theorem 1 we present the distribution properties of $\hat{R}_{G M V}, \hat{V}_{G M V}$, and $\hat{s}$.

TheOREM 1. Let $\boldsymbol{X}=\left(\boldsymbol{x}_{1}, \ldots, \boldsymbol{x}_{n}\right) \sim \mathcal{M E D}_{k}(\boldsymbol{\mu}, \boldsymbol{\Sigma}, g()$.$) . Assume that \boldsymbol{\Sigma}$ is positive definite. Let $k>2$ and $n>k$. Then it holds that

a) Given $r=r_{0}, \hat{V}_{G M V}$ is independent of $\left(\hat{R}_{G M V}, \hat{s}\right)$.

b) $(n-1) \hat{V}_{G M V} / V_{G M V} \mid r=r_{0} \sim r_{0}^{2} \chi_{n-k}^{2}$.

c) $\frac{n(n-k+1)}{(n-1)(k-1)} \hat{s} \mid r=r_{0} \sim F_{k-1, n-k+1, n s / r_{0}^{2}}$.

d) $\hat{R}_{G M V} \mid \hat{s}=y, r=r_{0} \sim \mathcal{N}\left(R_{G M V}, \frac{1+n /(n-1) y}{n} V_{G M V} r_{0}^{2}\right)$.

e) The joint density function is given by

$$
\begin{aligned}
f_{\hat{R}_{G M V},}, \hat{V}_{G M V}, \hat{s} & (x, z, y) \\
= & \int_{0}^{\infty} \frac{n(n-k+1)}{(k-1) V_{G M V} r_{0}^{2}} f_{N\left(R_{G M V}, \frac{1+n /(n-1) y}{n} V_{G M V} r_{0}^{2}\right)}(x) \\
& \times f_{\chi_{n-k}^{2}}\left(\frac{n-1}{V_{G M V} r_{0}^{2}} z\right) f_{F_{k-1, n-k+1, n s / r_{0}^{2}}}\left(\frac{n(n-k+1)}{(n-1)(k-1)} y\right) f_{r}\left(r_{0}\right) d r_{0} .
\end{aligned}
$$

Proof. Given $r=r_{0}$ it holds that the $\boldsymbol{x}_{i}$ 's are independently distributed with $\boldsymbol{x}_{i} \mid r=r_{0} \sim \mathcal{N}\left(\boldsymbol{\mu}, r_{0}^{2} \boldsymbol{\Sigma}\right)$. Application of Lemma 1 of Bodnar and Schmid (2009) leads to the statement of the theorem. The theorem is proved.

\subsection{Confidence region for the efficient frontier}

A joint test for three characteristics of the efficient frontier is given by

$$
H_{0}: R_{G M V}=R_{0}, \quad V_{G M V}=V_{0}, \quad s=s_{0}
$$


against

$$
H_{1}: R_{G M V}=R_{1} \neq R_{0} \quad \text { or } \quad V_{G M V}=V_{1} \neq V_{0} \quad \text { or } \quad s=s_{1} \neq s_{0} .
$$

For testing (3.5) we use the results of Theorem 1, which motivate the application of the test statistic $T=\left(T_{R}, T_{V}, T_{S}\right)^{\prime}$ with

$$
\begin{aligned}
& T_{R}=\sqrt{n} \frac{\hat{R}_{G M V}-R_{0}}{\sqrt{V_{0}} \sqrt{1+n /(n-1) \hat{\boldsymbol{\mu}}^{\prime} \hat{\boldsymbol{Q}} \hat{\boldsymbol{\mu}}}}, \\
& T_{V}=(n-1) \frac{\hat{V}_{G M V}}{V_{0}}, \\
& T_{S}=\frac{n(n-k+1)}{(k-1)(n-1)} \hat{\boldsymbol{\mu}}^{\prime} \hat{\boldsymbol{Q}} \hat{\boldsymbol{\mu}} .
\end{aligned}
$$

Similar test statistic was considered by Bodnar and Schmid (2009) in the normal case. The distribution of $T$ is derived in Theorem 2 under the null and alternative hypotheses.

TheOREM 2. Let $\boldsymbol{X}=\left(\boldsymbol{x}_{1}, \ldots, \boldsymbol{x}_{n}\right) \sim \mathcal{M E D}_{k}(\boldsymbol{\mu}, \boldsymbol{\Sigma}, g()$.$) . Assume that \boldsymbol{\Sigma}$ is positive definite. Let $k>2$ and $n>k$. Then it holds that

a) Let $R_{G M V}=R_{1}, V_{G M V}=V_{1}$, and $s=s_{1}$. The density of $T$ is given by

$$
\begin{aligned}
f_{T}(x, y, z)= & \eta^{-1} \int_{0}^{\infty} r_{0}^{-2} f_{\chi_{n-k}^{2}}\left(\frac{z}{r_{0}^{2} \eta}\right) f_{N\left(\sqrt{\eta} \delta(y), r_{0}^{2} \eta\right)}(x) \\
& \times f_{F_{k-1, n-k+1, n s_{1} / r_{0}^{2}}}(y) f_{r}\left(r_{0}\right) d r_{0},
\end{aligned}
$$

with $\delta(y)=\sqrt{n} \lambda_{1} / \sqrt{1+\frac{k-1}{n-k+1} y}, \eta=V_{1} / V_{0}, \lambda_{1}=\left(R_{1}-R_{0}\right) / \sqrt{V_{1}}$, and $s_{1}=$ $\boldsymbol{\mu}^{\prime} \boldsymbol{Q} \boldsymbol{\mu}$.

b) Under the null hypothesis the density of $T$ under $H_{0}$ is given by

$$
\begin{aligned}
f_{T}(x, y, z)= & \int_{0}^{\infty} r_{0}^{-2} f_{\chi_{n-k}^{2}}\left(z / r_{0}^{2}\right) f_{N\left(0, r_{0}^{2}\right)}(x) \\
& \times f_{F_{k-1, n-k+1, n s_{0} / r_{0}^{2}}}(y) f_{r}\left(r_{0}\right) d r_{0} .
\end{aligned}
$$

Proof. The proof of Theorem 2 follows from Proposition 3 of Bodnar and Schmid (2009) by considering first the conditional distribution of $\boldsymbol{X}$ given $r=r_{0}$ and then integrating over $r_{0}$. The theorem is proved.

The results of Theorem 2 are used to derive the power function of the test for (3.5) which depends on $\boldsymbol{\mu}$ and $\boldsymbol{\Sigma}$ only through the quantities $\eta, \lambda_{1}$, and $s_{1}$. The power function is equal to

$$
\begin{aligned}
G_{T ; \alpha}\left(\eta, \lambda_{1}, s_{1}\right)=1 & -\int_{0}^{\infty}\left(1-G_{T_{V} ; \tilde{\alpha}}(\eta)\right) \\
& \times \int_{z_{\tilde{\alpha} / 2}}^{z_{1-\tilde{\alpha} / 2}} \int_{s_{\tilde{\alpha} / 2}}^{s_{1-\tilde{\alpha} / 2}} f_{N\left(\sqrt{\eta} \delta(y), \eta r_{0}^{2}\right)}(x) \\
& \times f_{F_{k-1, n-k+1, n s_{1} / r_{0}^{2}}}(y) f_{r}\left(r_{0}\right) d y d x d r_{0},
\end{aligned}
$$


where

$$
G_{T_{V} ; \tilde{\alpha}}(\eta)=1-F_{\chi_{n-k}^{2}}\left(\frac{\chi_{n-k ; 1-\tilde{\alpha} / 2}^{2}}{\eta r_{0}^{2}}\right)+F_{\chi_{n-k}^{2}}\left(\frac{\chi_{n-k ; \tilde{\alpha} / 2}^{2}}{\eta r_{0}^{2}}\right)
$$

and $1-\alpha=(1-\tilde{\alpha})^{3}$, i.e. $\tilde{\alpha}=1-\sqrt[3]{1-\alpha}$. The quantities $s_{\tilde{\alpha} / 2}$ and $s_{1-\tilde{\alpha} / 2}$ are the lower and upper bounds of the $(1-\tilde{\alpha})$-confidence interval for $s$.

In Figures 1 and 2, we present (3.11) as a function of $\eta$ and $\lambda_{1}$ in the case of the matrix $t$-distribution with 5 degrees of freedom and in the case of the matrix $t$-distribution with 15 degrees of freedom. The figures show that the test for (3.5) is more powerful when the matrix $t$-distribution with larger number of degrees of freedom, i.e. with the smaller tails, is considered.

Next, we construct the joint confidence set for the three characteristics of the efficient frontier. From Theorem 2, it follows that, given $r=r_{0}$, the statistics $T_{R}$, $T_{V}$, and $T_{S}$ are mutually independent. This fact simplifies the construction of the confidence region. In order to account for the uncertainty of the generating variable $r$, we put $1-\alpha=\left(1-\alpha^{*}\right)^{4}$, e.g. $\alpha^{*}=1-\sqrt[4]{1-\alpha}$. Using the fact that for given $r=r_{0} \boldsymbol{x}_{i}$ 's are independently distributed with $\boldsymbol{x}_{i} \mid r=r_{0} \sim \mathcal{N}\left(\boldsymbol{\mu}, r_{0}^{2} \boldsymbol{\Sigma}\right)$, the simultaneous confidence set $\mathcal{A}\left(r_{0}\right)$ consists of all points $\left(R_{G M V}, V_{G M V}, s\right)$ that satisfy

$$
\left(R_{G M V}-\hat{R}_{G M V}\right)^{2} \leq z_{1-\alpha^{*} / 2}^{2}\left(\frac{1}{n}+\frac{\hat{s}}{n-1}\right) V_{G M V} r_{0}^{2}
$$

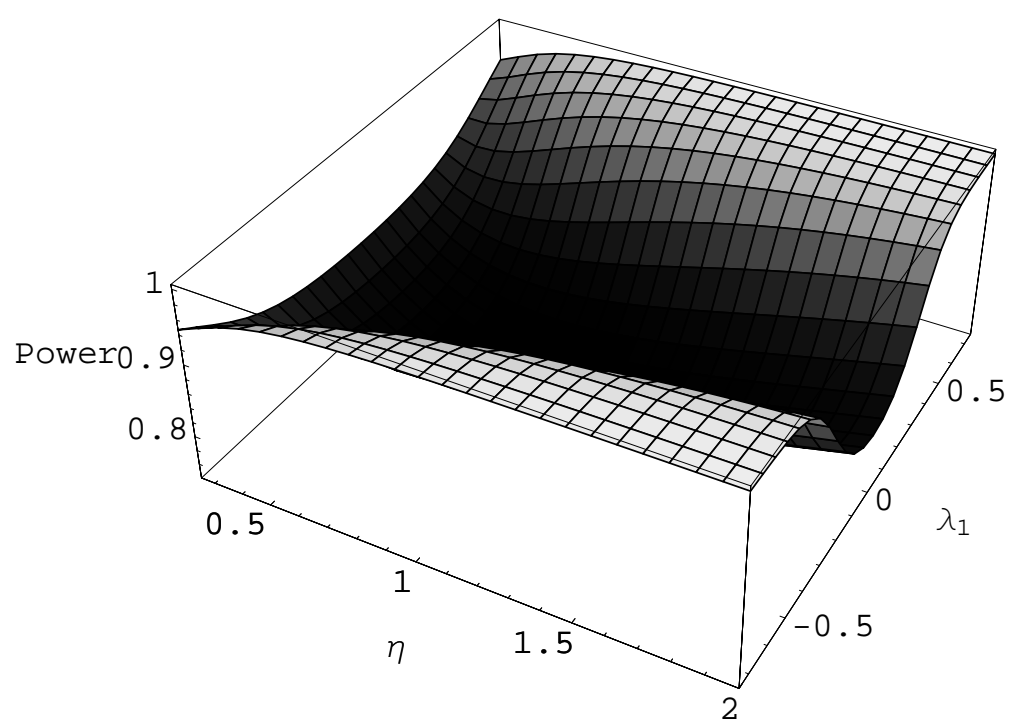

Figure 1. Power of the test based on $T$ for testing problem (3.5) as a function of $\eta=V_{1} / V_{0}$ and $\lambda_{1}=\left(R_{1}-R_{0}\right) / \sqrt{V_{1}}$ (data of Section $4, s_{1}=0.224157, n=60, k=5$, and $\alpha=5 \%$ ). The asset returns are assumed to be matrix $t$-distributed with 5 degrees of freedom. 


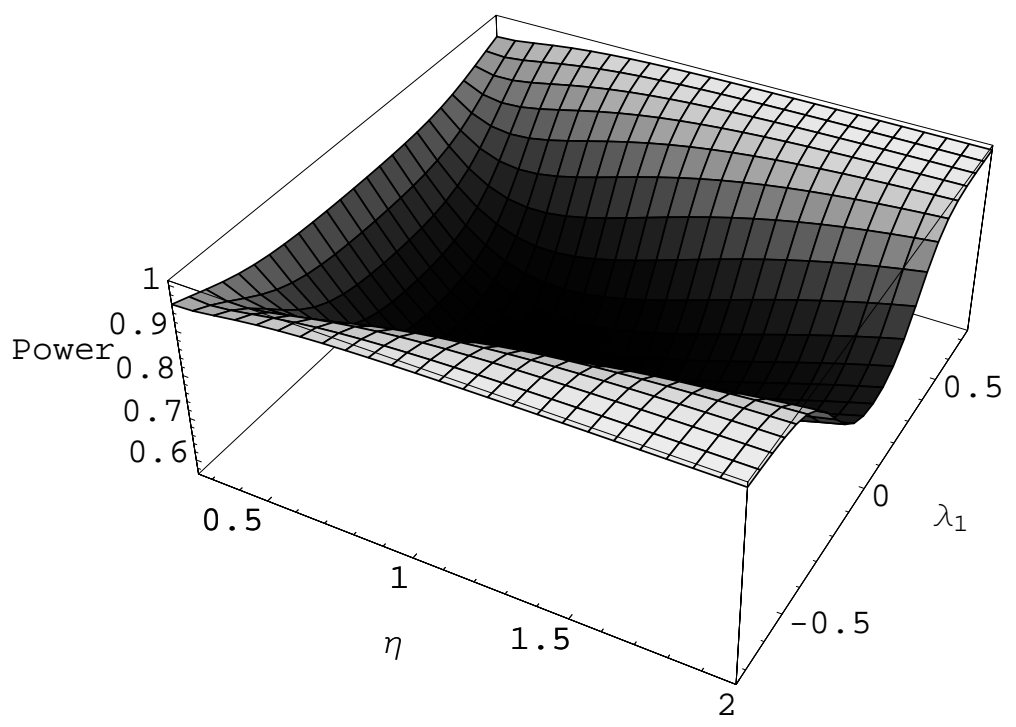

Figure 2. Power of the test based on $T$ for testing problem (3.5) as a function of $\eta=V_{1} / V_{0}$ and $\lambda_{1}=\left(R_{1}-R_{0}\right) / \sqrt{V_{1}}$ (data of Section $4, s_{1}=0.224157, n=60, k=5$, and $\alpha=5 \%$ ). The asset returns are assumed to be matrix $t$-distributed with 15 degrees of freedom.

$$
\begin{aligned}
& V_{G M V} \in\left[\frac{(n-1) \hat{V}_{G M V}}{r_{0}^{2} \chi_{n-k ; 1-\alpha^{*} / 2}^{2}}, \frac{(n-1) \hat{V}_{G M V}}{r_{0}^{2} \chi_{n-k ; \alpha^{*} / 2}^{2}}\right]=\left[\hat{V}_{l}, \hat{V}_{u}\right], \\
& r_{0}^{2} \hat{s}_{\alpha^{*} / 2} \leq s \leq r_{0}^{2} \hat{s}_{1-\alpha^{*} / 2} .
\end{aligned}
$$

The confidence interval for $s$ is obtained as a confidence interval for the noncentrality parameter of the noncentral $F$-distribution (see Lam (1987)).

Let $F_{R}\left(r_{\max }\right)=1-\alpha^{*} / 2$ and $F_{R}\left(r_{\min }\right)=\alpha^{*} / 2$. Then the confidence region for the efficient frontier is defined as the border of the set $\left\{\mathcal{A}(r): r_{\min } \leq r \leq\right.$ $\left.r_{\max }\right\}$, which is given by

$$
\begin{aligned}
& \left(R_{G M V}-\hat{R}_{G M V}\right)^{2} \leq z_{1-\alpha^{*} / 2}^{2}\left(\frac{1}{n}+\frac{\hat{s}}{n-1}\right) V_{G M V} r_{\max }^{2} \\
& \text { for } R_{G M V}>\hat{R}_{G M V} \text {, } \\
& \left(R_{G M V}-\hat{R}_{G M V}\right)^{2} \leq z_{1-\alpha^{*} / 2}^{2}\left(\frac{1}{n}+\frac{\hat{s}}{n-1}\right) V_{G M V} r_{\min }^{2} \\
& \text { for } R_{G M V}<\hat{R}_{G M V} \text {, } \\
& V_{G M V} \in\left[\frac{(n-1) \hat{V}_{G M V}}{r_{\max }^{2} \chi_{n-k ; 1-\alpha^{*} / 2}^{2}}, \frac{(n-1) \hat{V}_{G M V}}{r_{\min }^{2} \chi_{n-k ; \alpha^{*} / 2}^{2}}\right]=\left[\hat{V}_{l}, \hat{V}_{u}\right] \\
& r_{\min }^{2} \hat{s}_{\alpha^{*} / 2} \leq s \leq r_{\max }^{2} \hat{s}_{1-\alpha^{*} / 2} .
\end{aligned}
$$

We denote this set by $\mathcal{A}^{*}$. Because the efficient frontier lies in the mean-variance space, it would be interesting to derive the expression for $\mathcal{A}^{*}$ in the mean-variance 
space. Note, that the confidence region for the efficient frontier consists of all parabolas $\left(R-R_{G M V}\right)^{2}=s\left(V-V_{G M V}\right)$, where $R_{G M V}$ and $V_{G M V}$ satisfy (3.12)(3.14) and $s$ satisfies (3.15). It can be expressed as

$$
\mathcal{B}=\left\{\left(R-R_{G M V}\right)^{2}=s\left(V-V_{G M V}\right): \quad\left(R_{G M V}, V_{G M V}, s\right) \in \mathcal{A}^{*}\right\} .
$$

Let

$$
\begin{aligned}
& g_{l}=z_{1-\alpha^{*} / 2} \sqrt{1+\frac{n \hat{s}}{n-1}} \frac{\sqrt{n-1} \sqrt{\hat{V}_{G M V}}}{\sqrt{n \chi_{n-k ; 1-\alpha^{*} / 2}^{2}}}, \\
& g_{u}=z_{1-\alpha^{*} / 2} \sqrt{1+\frac{n \hat{s}}{n-1}} \frac{\sqrt{n-1} \sqrt{\hat{V}_{G M V}}}{\sqrt{n \chi_{n-k ; \alpha^{*} / 2}^{2}}} \frac{r_{\max }}{r_{\min }} \\
& t=\frac{1}{1+z_{1-\alpha^{*} / 2}^{-2} \hat{s}_{1-\alpha^{*} / 2} \frac{n(n-1)}{n(1+\hat{s})-1}} .
\end{aligned}
$$

Using the proof of Theorem 1 of Bodnar and Schmid (2009), we get the expression of $\mathcal{B}$.

THEOREM 3. It holds that $\mathcal{B}$ is equal to the set of all pairs $(R, V)$ satisfying all of the following conditions

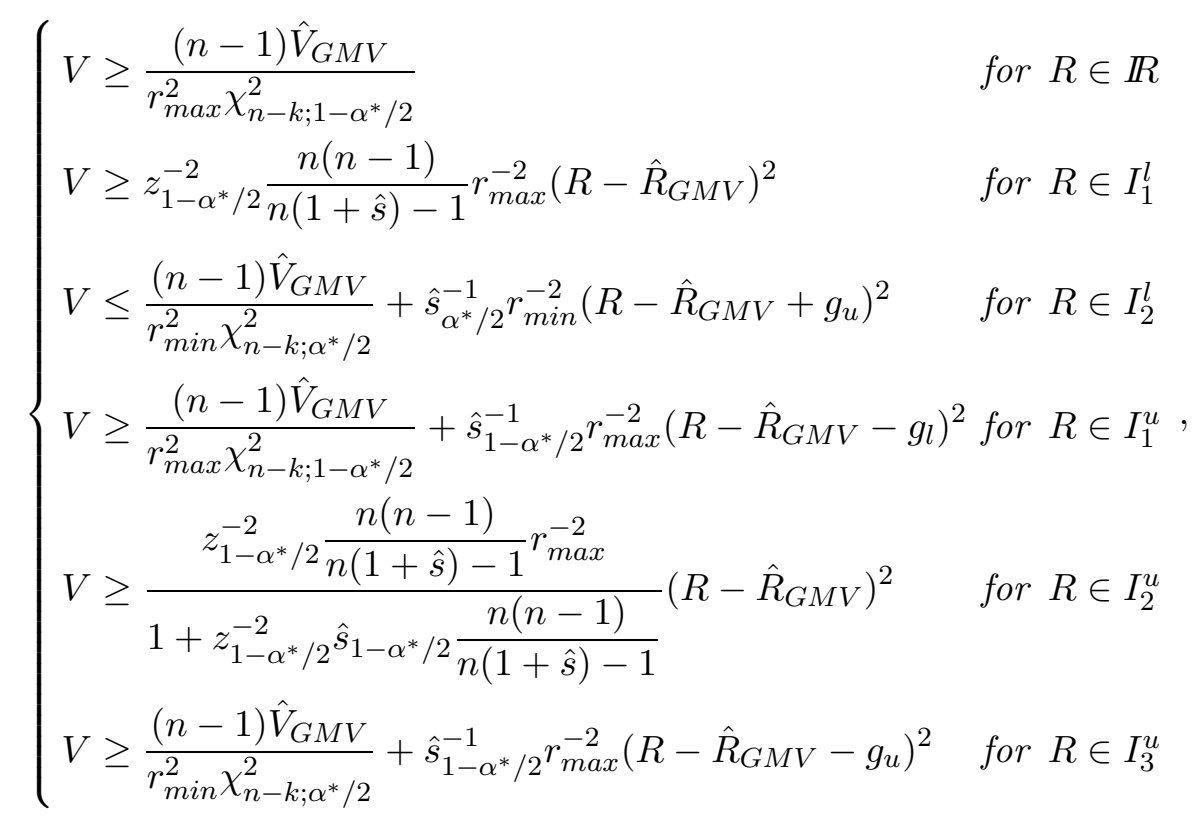

where $I_{1}^{u}=\left[\hat{R}_{G M V}+g_{l}, \hat{R}_{G M V}+g_{l} / t\right], I_{2}^{u}=\left[\hat{R}_{G M V}+g_{l} / t, \hat{R}_{G M V}+g_{u} / t\right], I_{3}^{u}=$ $\left(\hat{R}_{G M V}+g_{u} / t, \infty\right), I_{1}^{l}=\left[\hat{R}_{G M V}-g_{u}, \hat{R}_{G M V}-g_{l}\right]$, and $I_{2}^{l}=\left[\hat{R}_{G M V}-g_{u},+\infty\right)$. 


\subsection{Unbiased estimator of the efficient frontier}

Basak et al. (2005) and Siegel and Woodgate (2007) showed that the sample efficient frontier is overly optimistic and overestimates the true location of the efficient frontier in the mean-variance space. Bodnar and Bodnar (2009) corrected the overoptimism of the sample efficient frontier by deriving the unbiased estimator of the efficient frontier assuming the asset returns to be normally distributed. In Theorem 4, we extend this result by assuming the asset returns to follow a matrix variate mixture of normal distributions.

TheOREM 4. Let $\boldsymbol{X}=\left(\boldsymbol{x}_{1}, \ldots, \boldsymbol{x}_{n}\right) \sim \mathcal{M E D}_{k}(\boldsymbol{\mu}, \boldsymbol{\Sigma}, g()$.$) . Assume that \boldsymbol{\Sigma}$ is positive definite. Let $k>2$ and $n>k$. Let $E\left(r^{2}\right)$ and $E\left(r^{-2}\right)$ exist. Then the unbiased estimator of the efficient frontier

$$
\psi(R, V)=\left(R-R_{G M V}\right)^{2}-\frac{1}{E\left(r^{2}\right)} s\left(V-E\left(r^{2}\right) V_{G M V}\right)
$$

is given by

$$
\begin{aligned}
\psi_{u}(R, V)= & \left(R-\hat{R}_{G M V}\right)^{2}-\frac{(n-2)(n-1)}{n(n-k)(n-k-1)} \hat{V}_{G M V}-\frac{1}{E\left(r^{2}\right) E\left(r^{-2}\right)} \\
& \times\left(\frac{n-k-1}{n-1} \hat{s}-\frac{k-1}{n}\right) \\
& \times\left(V-E\left(r^{2}\right) E\left(r^{-2}\right) \frac{(n-k-2)(n-1)}{(n-k-1)(n-k)} \hat{V}_{G M V}\right) .
\end{aligned}
$$

Proof. Consider

$$
\begin{aligned}
\mathrm{E}\left(\psi_{u}(R, V)\right)= & \mathrm{E}\left(\mathrm{E}\left(\psi_{u}(R, V) \mid r\right)\right) \\
= & \mathrm{E}\left(\mathrm{E}\left(\left(R-\hat{R}_{G M V}\right)^{2} \mid r\right)\right)-\frac{(n-2)(n-1)}{n(n-k)(n-k-1)} \mathrm{E}\left(\mathrm{E}\left(\hat{V}_{G M V} \mid r\right)\right) \\
& -\frac{1}{\mathrm{E}\left(r^{2}\right) \mathrm{E}\left(r^{-2}\right)} \mathrm{E}\left(\left(\frac{n-k-1}{n-1} \mathrm{E}(\hat{s} \mid r)-\frac{k-1}{n}\right)\right. \\
& \left.\times\left(V-\mathrm{E}\left(r^{2}\right) \mathrm{E}\left(r^{-2}\right) \frac{(n-k-2)(n-1)}{(n-k-1)(n-k)} \mathrm{E}\left(\hat{V}_{G M V} \mid r\right)\right)\right),
\end{aligned}
$$

where in the last equality we use that $\hat{s}$ and $\hat{V}_{G M V}$ are independent given $r$ (see Theorem 1).

Now from Theorem 1, we obtain

$$
\begin{aligned}
\mathrm{E}(\mathrm{E} & \left.\left(\left(R-\hat{R}_{G M V}\right)^{2} \mid r\right)\right) \\
& =\left(R-R_{G M V}\right)^{2}+\mathrm{E}\left(\operatorname{Var}\left(\hat{R}_{G M V} \mid r\right)\right) \\
& =\left(R-R_{G M V}\right)^{2}+\operatorname{Var}\left(\mathrm{E}\left(\hat{R}_{G M V} \mid \hat{s}, r\right)\right)+\mathrm{E}\left(\operatorname{Var}\left(\hat{R}_{G M V} \mid \hat{s}, r\right)\right) \\
& =\left(R-R_{G M V}\right)^{2}+\operatorname{Var}\left(R_{G M V}\right)+\mathrm{E}\left(\left(\frac{1}{n}+\frac{1}{n-1} \mathrm{E}(\hat{s} \mid r)\right) V_{G M V} r^{2}\right) \\
& =\left(R-R_{G M V}\right)^{2}+\mathrm{E}\left(\left(\frac{n-2}{n(n-k-1)}+\frac{1}{n-k-1} \frac{s}{r^{2}}\right) V_{G M V} r^{2}\right) .
\end{aligned}
$$


The last equality follows from the fact that (see Johnson et al. (1995, p. 481))

$$
\mathrm{E}(\hat{s} \mid r)=\frac{n-1}{n-k-1} \frac{s}{r^{2}}+\frac{(n-1)(k-1)}{n(n-k-1)} .
$$

Hence,

$$
\begin{aligned}
\mathrm{E}\left(\mathrm{E}\left(\left(R-\hat{R}_{G M V}\right)^{2} \mid r\right)\right)= & \left(R-R_{G M V}\right)^{2}+\frac{(n-2) \mathrm{E}\left(r^{2}\right) V_{G M V}}{n(n-k-1)} \\
& +\frac{s V_{G M V}}{n-k-1} .
\end{aligned}
$$

Application of (3.22) and

$$
\mathrm{E}\left(\hat{V}_{G M V} \mid r\right)=\frac{n-k}{n-1} r^{2} V_{G M V}
$$

leads to

$$
\begin{aligned}
\frac{1}{\mathrm{E}\left(r^{2}\right) \mathrm{E}\left(r^{-2}\right)} \mathrm{E}( & \left(\frac{n-k-1}{n-1} \mathrm{E}(\hat{s} \mid r)-\frac{k-1}{n}\right) \\
& \left.\times\left(V-\mathrm{E}\left(r^{2}\right) \mathrm{E}\left(r^{-2}\right) \frac{(n-k-2)(n-1)}{(n-k-1)(n-k)} \mathrm{E}\left(\hat{V}_{G M V} \mid r\right)\right)\right) \\
= & \frac{1}{\mathrm{E}\left(r^{2}\right) \mathrm{E}\left(r^{-2}\right)} s \mathrm{E}\left(r^{-2}\left(V-\mathrm{E}\left(r^{2}\right) \mathrm{E}\left(r^{-2}\right) \frac{n-k-2}{n-k-1} V_{G M V} r^{2}\right)\right) \\
= & \frac{1}{\mathrm{E}\left(r^{2}\right)} s\left(V-\mathrm{E}\left(r^{2}\right) \frac{n-k-2}{n-k-1} V_{G M V}\right) .
\end{aligned}
$$

From (3.24) we get

$$
\frac{(n-2)(n-1)}{n(n-k)(n-k-1)} \mathrm{E}\left(\mathrm{E}\left(\hat{V}_{G M V} \mid r\right)\right)=\frac{n-2}{n(n-k-1)} \mathrm{E}\left(r^{2}\right) V_{G M V} .
$$

Putting (3.23), (3.25), and (3.26) together, we obtain

$$
\mathrm{E}\left(\psi_{u}(R, V)\right)=\left(R-R_{G M V}\right)^{2}-\frac{1}{\mathrm{E}\left(r^{2}\right)} s\left(V-\mathrm{E}\left(r^{2}\right) V_{G M V}\right)=0 .
$$

The last equality completes the proof of the theorem.

Although for determining the population efficient frontier $\mathrm{E}\left(r^{2}\right)$ need only exist, for constructing an unbiased estimator both the moments $\mathrm{E}\left(r^{2}\right)$ and $\mathrm{E}\left(r^{-2}\right)$ are used.

\subsection{Overall $\boldsymbol{F}$-test}

In Section 2, it was shown that if $\gamma=\infty$ the efficient frontier degenerates into a straight line. If $s=0$ the efficient frontier is also a straight line without imposing any assumption on $\gamma$. In both cases, there is only one optimal portfolio, namely the GMV portfolio. Assuming the asset returns to be matrix elliptically 
contoured distributed Bodnar and Schmid (2008a) derived an exact test on the weight of the GMV portfolio, while Bodnar (2007) considered sequential procedures for monitoring the weights of the GMV portfolio.

Next, we consider a test for testing $s=0$. The test hypothesis is given by

$$
H_{0}: s=0 \text { against } H_{1}: s>0 \text {. }
$$

For testing (3.27) we use the results of Theorem 1. Because the non-central $F$-distribution with $s=0$ is a central $F$-distribution, it holds that the null hypothesis is rejected if

$$
T_{S}=\frac{n(n-k+1)}{(n-1)(k-1)} \hat{s}>F_{k-1, n-k+1 ; 1-\alpha} .
$$

$F_{k-1, n-k+1 ; 1-\alpha}$ denotes the $(1-\alpha)$-quantile of the central $F$-distribution with $k-1$ and $n-k+1$ degrees of freedom. Note, that the distribution of the test statistic (3.28) does not depend on the distribution assumption imposed on the asset returns within the class of matrix elliptically contoured distributions. Thus, the test can easily be carried out using the the $(1-\alpha)$-quantile of the central F-distribution.

\section{Empirical illustration}

In order to get a better understanding for the results presented in Section 3 we consider an example with real data in this section. We make use of monthly data from Morgan Stanley Capital International for the equity market returns of

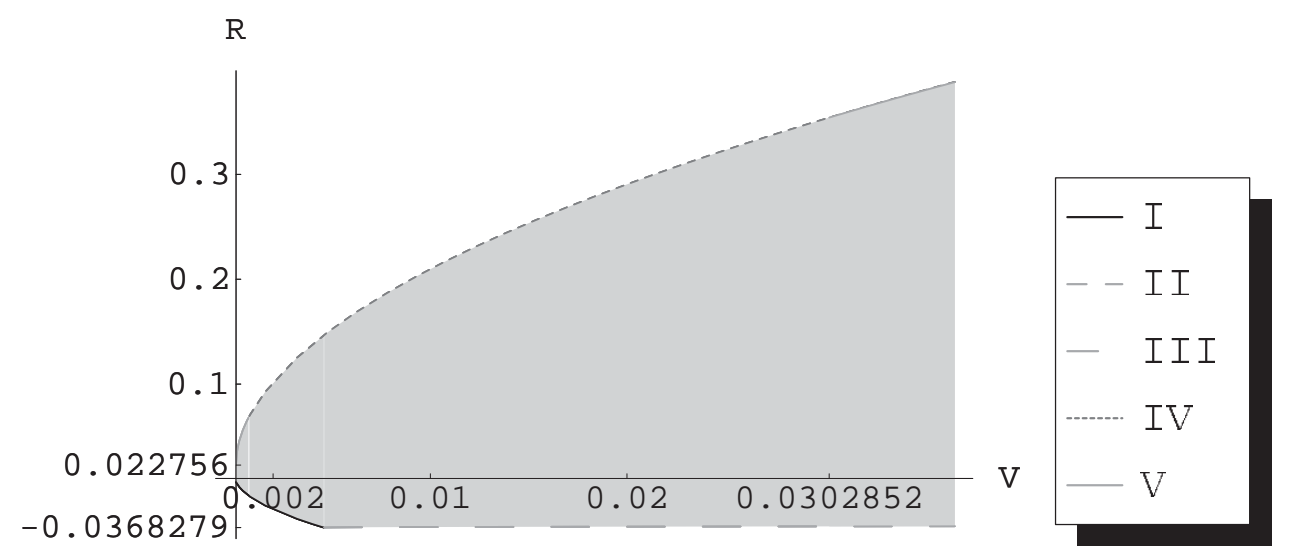

Figure 3. Geometric structure of the $85 \%$-confidence region for the efficient frontier in the mean-variance space (monthly data of Section 4, see (4.), shaded area $\mathcal{B}^{*}$ ). The estimated parameters of the efficient frontier are given by $\hat{R}_{G M V}=0.0145664, \hat{V}_{G M V}=0.0010337$, and $\hat{s}=0.224157$. The asset returns are assumed to be matrix $t$-distributed with 5 degrees of freedom. 
five developed countries (UK, Germany, USA, Canada, and Switzerland) for the period from July 1994 to June 1999. The parameters of the efficient frontier are estimated by

$$
\text { (4.1) } \quad \hat{R}_{G M V}=0.0145664, \quad \hat{V}_{G M V}=0.0010337, \quad \text { and } \quad \hat{s}=0.221457 .
$$

It holds that $k=5$ and $n=60$. We put $\alpha=0.15$, i.e. $\alpha^{*}=0.0 .0398$, $\hat{s}_{\alpha^{*} / 2}=0.000133, \hat{s}_{1-\alpha^{*} / 2}=0.4849, z_{1-\alpha^{*} / 2}=2.05566, \chi_{n-k ; \alpha^{*} / 2}^{2}=35.64$, and $\chi_{n-k ; 1-\alpha^{*} / 2}^{2}=78.64$. Next we insert these values in Theorem 3 to obtain the $85 \%$ confidence region of the efficient frontier in the mean-variance space which is bordered by five parabolas. When the matrix of the asset returns is assumed to be $t$-distributed with 5 degrees of freedom, it is given by

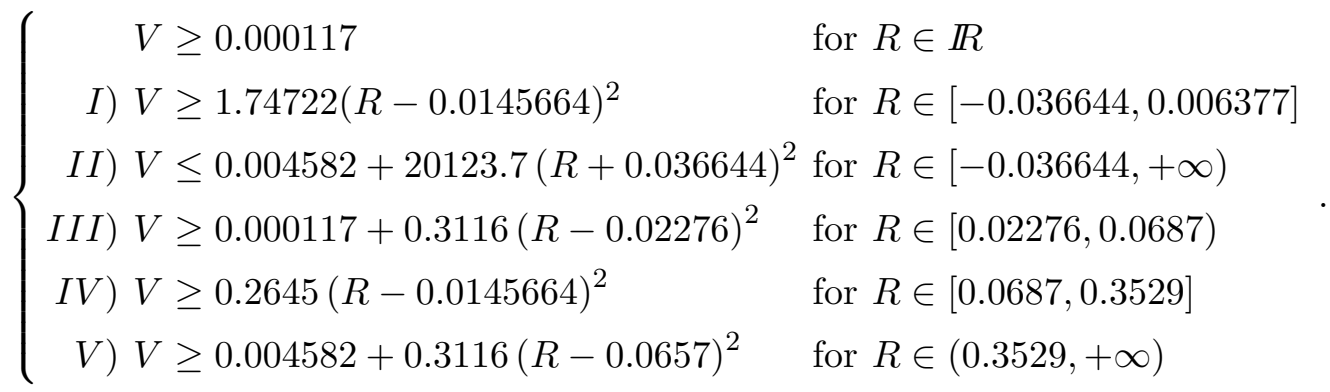

In case of the matrix $t$-distribution with 15 degrees of freedom, we get the following expression of the the $85 \%$ confidence region of the efficient frontier

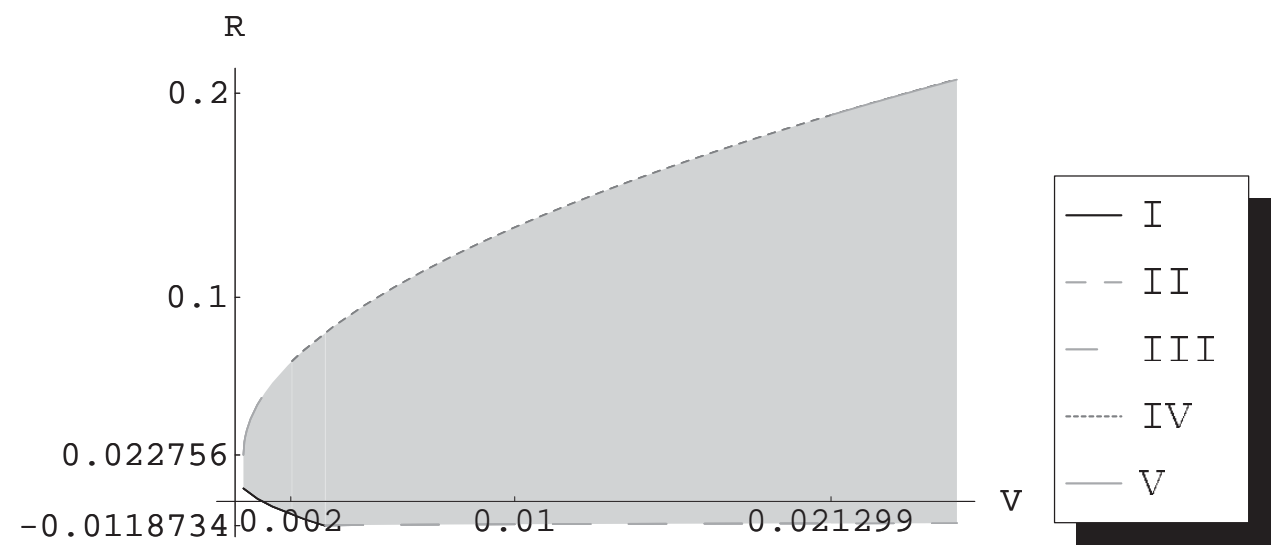

Figure 4. Geometric structure of the $85 \%$-confidence region for the efficient frontier in the mean-variance space (monthly data of Section 4, see (4.), shaded area $\mathcal{B}^{*}$ ). The estimated parameters of the efficient frontier are given by $\hat{R}_{G M V}=0.0145664, \hat{V}_{G M V}=0.0010337$, and $\hat{s}=0.224157$. The asset returns are assumed to be matrix $t$-distributed with 15 degrees of freedom. 


$$
\left\{\begin{array}{rlrl}
V \geq 0.000309 & & \text { for } R \in \mathbb{R} \\
I) & V \geq 4.61(R-0.0145664)^{2} & & \text { for } R \in[-0.01191,0.006377] \\
I I) & V \leq 0.003224+14156.6(R+0.01191)^{2} & & \text { for } R \in[-0.01191,+\infty) \\
I I I) & V \geq 0.000309+0.8222(R-0.02276)^{2} & & \text { for } R \in[0.02276,0.0687) \\
I V) & V \geq 0.69776(R-0.0145664)^{2} & & \text { for } R \in[0.0687,0.1895] \\
V) & V \geq 0.003224+0.8222(R-0.041)^{2} & & \text { for } R \in(0.0939,+\infty)
\end{array} .\right.
$$

The geometrical structure of both the confidence regions are shown in Figures 3 and 4 . We observe that the area of the confidence region is smaller when the asset returns are assumed to be matrix $t$-distributed with 15 degrees of freedom than in the case of the matrix $t$-distribution with 5 degrees of freedom. There are also a number of portfolios with negative expected returns in Figure 3 that belong to the confidence region. This set is much larger than the one given in Figure 4 .

\section{Acknowledgements}

The authors are thankful to the referee and the Editor for their suggestions which have improved the presentation in the paper.

\section{REFERENCES}

Basak, G. K., Jagannathan, R. and Ma, T. (2005). Estimation the Risk in Sample Efficient Portfolios, Working Paper, Northwestern University.

Berk, J. B. (1997). Necessary conditions for the CAPM, J. Econ. Theory, 73, 245-257.

Bodnar, O. (2007). Sequential procedures for monitoring covariances of asset returns, in $A d$ vances in Risk Management (ed. G. N. Gregoriou), Palgrave, London, 241-264.

Bodnar, O. and Bodnar, T. (2009). On the unbiased estimator of the efficient frontier, Quant. Financ. (under revision).

Bodnar, T. and Schmid, W. (2007). The distribution of the sample variance of the global minimum variance portfolio in elliptical models, Statistics, 41, 65-75.

Bodnar, T. and Schmid, W. (2008a). A test for the weights of the global minimum variance portfolio in an elliptical model, Metrika, 67, 127-143.

Bodnar, T. and Schmid, W. (2008b). Estimation of optimal portfolio compositions for gaussian returns, Statistics \& Decisions, 26, 179-201.

Bodnar, T. and Schmid, W. (2009). Econometrical analysis of the sample efficient frontier, Eur. J. Financ., 15, 317-335.

Bollerslev, T. (1986). Generalized autoregressive conditional heteroscedasticity, J. Econ., 31, $307-327$.

Chamberlain, G. A. (1983). A characterization of the distributions that imply mean-variance utility functions, J. Econ. Theory, 29, 185-201.

Chen, J. T., Gupta, A. K. and Troskie, C. G. (2003). The distribution of stock returns when the market is up, Commun. Statist. Theory, 32, 1541-1558.

Engle, R. F. (1982). Autoregressive conditional heteroscedasticity with estimates of the variance of U.K. inflation, Econometrica, 50, 987-1008.

Engle, R. F. (2002). Dynamic conditional correlation - a simple class of multivariate GARCH models, J. Bus. Econ. Statist., 20, 339-350.

Fang, K. T. and Zhang, Y. T. (1990). Generalized Multivariate Analysis, Springer-Verlag, Berlin and Science Press, Beijing. 
Fama, E. F. (1965). The behavior of stock market prices, J. Bus., 38, 34-105.

Gibbons, M. R., Ross, S. A. and Shanken, J. (1989). A test of the efficiency of a given portfolio, Econometrica, 57, 1121-1152.

Gupta, A. K. and Varga, T. (1993). Elliptically Contoured Models in Statistics, Kluwer Academic Publishers.

Hodgson, D. J., Linton, O. and Vorkink, K. (2002). Testing the capital asset pricing model efficiency under elliptical symmetry: a semiparametric approach, J. Appl. Econ., 17, 617639.

Jobson, J. D. (1991). Confidence region for the mean-variance efficient set: an alternative approach to estimation risk, Rev. Quant. Financ. Acc., 1, 235-257.

Johnson, N. L., Kotz, S. and Balakrishnan, N. (1995). Continuous Univariate Distributions, vol. 2, New York: Wiley.

Kan, R. and Smith, D. R. (2008). The distribution of the sample minimum-variance frontier, Manage. Sci., 54, 1364-1380.

Lam, Y.-M. (1987). Confidence limits for non-centrality parameters of noncentral chi-squared and F distributions, ASA Proceedings of the Statistical Computing Section, 441-443.

Markowitz, H. (1952). Portfolio selection, J. Financ., 7, 77-91.

Markowitz, H. (1991). Foundations of portfolio theory, J. Financ., 7, 469-477.

Merton, R. C. (1972). An analytical derivation of the efficient frontier, J. Financ. Quant. Anal., 7, 1851-1872.

Mittnik, S. and Rachev, S. T. (1993). Modelling asset returns with alternative stable distributions, Econ. Rev., 12, 261-330.

Nelson, D. (1991). Conditional heteroscedasticity in stock returns: a new approach, Econometrica, 59, 347-370.

Owen, J. and Rabinovitch, R. (1983). On the class of elliptical distributions and their applications to the theory of portfolio choice, J. Financ., 38, 745-752.

Siegel, A. F. and Woodgate, A. (2007). Performance of portfolios optimized with estimation error, Manage. Sci., 53, 1005-10015.

Zhou, G. (1993). Asset-pricing tests under alternative distributions, J. Financ., 48, 1927-1942. 